\title{
Very Mild Hallucination
}

National Cancer Institute

\section{Source}

National Cancer Institute. Very Mild Hallucination. NCI Thesaurus. Code C156241.

While resting or going to sleep, sees visions, smells odors or hears voices, sounds, or whispers in the absence of external stimulation, but no impairment in functioning. 